\title{
Statistical mechanics study of the thermodynamics of binary alloys with long-rang interaction
}

\author{
L.P.Danilova, Yu.P.Virchenko \\ Belgorod State University, 308015, Belgorod
}

Received February 25, 2018

\begin{abstract}
In frameworks of statistical mechanics of lattice gas model, it is calculated the free energy and the specific heat of binary alloys in the case when their atoms have a long-rang interaction. The calculation method is based on the use of connection between the statistical system of alloy atoms and the so-called lattice gas model. For the analysis of temperature dependence of each term in lattice gas virial expansion it is applied its decomposition on Ursell's function degree. Such an approximation method represents a modification of high temperature expansions of various model thermodynamic characteristics.
\end{abstract}

Keywords: binary alloy, lattice gas, interaction potential, virial expansion.

В рамках равновесной статистической механики вычисляется свободная энергия и удельная теплоемкость бинарных сплавов металлов, атомы которых обладают дальнодействующим потенциалом взаимодействия. Метод вычисления основан на использования связи между статистической системой атомов сплава и т.н. решеточным газом. Для анализа температурной зависимости членов вириального разложения решеточного газа применяется их разложение по степенмм вхождения функции Урселла. Такой метод приближений представляет собой модификацию высокотемпературных разложений термодинамических характеристик модели.

Термодінаміка бінарних сплавів із взаємодією, що має великий радіус, у рамках статистичного підходу. Л.П.Данілова, ЮО.П.Вірченко.

У рамках рівноважної статистичної механіки обчислюеться вільна енергія та питома теплосмність у бінарних металевих сплавах, атоми яких мають взасмодію с великим радіусом. Метод обчислення заснован на зв'язку між статистичною системою атомів сплаву та так званим гратковим газом. Для анализу температурної залежності членів віріального розкладу у моделі граткого газу застосовується розклад за ступенями входження функції Урселла. Такий метод уявляє собою модіфікацію високотемпературних розкладів термодинамічних характеристик моделі.

\section{Introduction}

Investigation of alloys thermodynamics in frameworks of statistical physics always attracted attentior. of theoreticians due to availability of structural phase transitions in such systems. In binary alloys, ir particular, it is observed phase transition of the «order-disorder» type when the temperature is decreasec (see, for example,[1], [2]). Such a transition is observed experimentally by the Bragg scattering of X-rays in crystal. Namely, in the ordered state, X-rays scattering detects the availability of two systems of atomic planes divided some distance. In contrary, in unordered state, there is only one system of scatterinc planes which are separated by doubly less distance. Besides, the transition has been accompanied a singularity in the temperature $T$ dependence of the heat heat $C_{V}(T)$ so, that it increases unboundedly wher temperature is approached to its critical value. 
Theoretical study of thermodynamics with the goal of setting of media thermodynamic characteristics dependencies on some control intensive thermodynamic parameters which are determined by media microscopic characteristics should be based with inevitability on the approach connected with application of equilibrium statistical mechanics methods. This approach is based on determination of each medium microscopic state energy, i.e. it should be defined by the hamiltonian of relevant physical system consisting of any large number of atoms. Usually (see [1], [2]), one is restricted to account only the interaction between nearest neighbors in crystal lattice, when the interaction between atoms of two binary alloy components is defined. Its magnitude is determined by lattice distribution of different atoms.

Let us consider the crystal sample $\Lambda$ that is described by the set of three-dimensional vectors $\mathbf{x}=n_{1} \mathbf{e}_{1}+n_{2} \mathbf{e}_{2}+n_{3} \mathbf{e}_{3}$, where $n_{1}, n_{2}, n_{3}$ are numbers $n_{j}=0,1,2, \ldots, L-1$. For simplicity, we suppose that crystal lattice constants are physically dimensionless and so they are equal to one.

We introduce the sign function $\sigma(\mathbf{z})$ on the crystal lattice. Such a function takes values \pm 1 in each lattice site $\mathbf{z}$. If there exist atoms of two kinds $\mathrm{A}$ and $\mathrm{B}$. Then, $\sigma(\mathbf{z})=1$, when the atom has the type $\mathrm{A}$ in the site $z$, and $\sigma(\mathbf{z})=-1$, when it has the type B in this site. Suppose that $V_{1,1}$ is equal to the interaction energy of two A atoms in nearest lattice sites. Similarly, $V_{-1,-1}$ is equal to the interaction energy of two $\mathrm{B}$ atoms and, correspondingly, $V_{1,-1}=V_{-1,1}$ is equal to the interaction energy of two different atoms, i.e. A and B kinds. Then, one may write down the model hamiltonian in the following form:

$$
\mathrm{H}[\sigma(\mathbf{z})]=\sum_{\{\mathbf{x}, \mathbf{y}:|\mathbf{x}-\mathbf{y}|=\min \}} V_{\sigma(\mathbf{x}), \sigma(\mathbf{y})}
$$

The sum in Eq. (1) is extened on all pairs $\{\mathbf{x}, \mathbf{y}\}$ of site radius vectors in the crystal lattice, which are located at the minimum distance. If $V_{1,-1}>\left(V_{11}+V_{-1,-1}\right) / 2$, two A atoms as well as two B atoms prefer to be near similar atoms. Conversely, if $V_{1,-1}<\left(V_{11}+V_{-1,-1}\right) / 2$, all atoms of the same kind repel apart each other and prefer to alternate its location in the lattice. Just, such a situation is realized at low temperatures. The ordered phase state of the medium arises. Atoms tends to be distributed by «the chess order» on the lattice.

Statistical study of the system containing a large number of metallic atoms of two kinds which are filled in crystal lattice and, consequently, the study of phase transition in such a system is based on the hamiltonian (1). It is done in a large number of theoretical researches. Usually, all such investigations are inevitably accomplished with application of some approximations with an accuracy that is poorly controlled. In particular, we note that the system with the hamiltonian (1) is reduced to so-called the Ising model. In three-dimensional case, there are not some explicit expressions based on standard special functions for temperature dependencies of its physical characteristics. Such an above-described situation leads to the use some approximations for the obtaining of concrete results. In connection with this situation, approximate methods acquire the special signification when we may control their accuracy. It determines their relevance.

Besides the general theoretical interest connected with the study of thermodynamics binary alloys with the view pointed out, there is also an interest connected with some applications, since, at last time, there are appeared some experimental data concerning with such alloys, which one may not describe on the basis of the statistical system with hamiltonian (1), when the interaction between nearest neighbors on the crystal lattice is taken into account only. In connection with such a situation, we do an attempt of constructing and research such a theoretical model wherein this drawback is eliminated.

In next section, we propose the hamiltonian of binary alloy when atoms have interaction with longrange radius, and we reduce the study of such a system to the so-called «lattice gas». Further, in the section 3, we apply the method of virial decomposition [3] that is known in statistical mechanics, in order to study thermodynamics of binary alloy with such a long-range interaction potential. At first, we find the explicit view of all expansion coefficients $c_{n}(T), n=2,3, \ldots$ It is done in the first approximation on Ursell's function degree. In section 4 we calculate the specific heat of binary alloy.

\section{Statistical mechanics system of binary alloy type}

We note that the model with hamiltonian (1), despite its great difficulty of correct mathematical research, is still very primitive from the view of binary alloy description. At least, it is connected with 
that interactions between atoms located on large distances is not taken into account in frames of such a system. However, lately, it is appeared theoretical interest to study some systems with such a character of interactions (see, for example, experimental works [4]-[7]).

For theoretical description of thermodynamics of above mentioned alloys in the framework of statistical mechanics, the hamiltonian (1) should be replaced presumably by the following:

$$
\mathrm{H}[\sigma]=\sum_{\{\mathbf{x}, \mathbf{y}\} \subset \Lambda} V_{\sigma(\mathbf{x}), \sigma(\mathbf{y})}(\mathbf{x}-\mathbf{y})
$$

where the sum is extended on all pairs of crystal sites in $\Lambda$, and introduced energies $V_{1,1}(\mathbf{z}), V_{-1,-1}(\mathbf{z})$, $V_{-1,1}(\mathbf{z})$ describe interactions between pairs of lattice sites with radius vectors $\mathbf{x}$ и $\mathbf{y}$. They depend only on the mutual spatial arrangement $\mathbf{z}=\mathbf{x}-\mathbf{y}$ of these sites. So, they do not change their meaning, if these sites are translated to an arbitrary vector. The sum in Eq.(2) applies to all pairs of the lattice sites of crystal sample with corresponding radius vectors $\mathbf{x}$ and $\mathbf{y}$. In this case, each random function $\sigma(\cdot)= \pm 1$ describes the state of lattice sites. Introduced functions $V_{\sigma, \sigma^{\prime}}(\mathbf{z})$ have the symmetry property: $V_{\sigma, \sigma^{\prime}}(-\mathbf{z})=V_{\sigma, \sigma^{\prime}}(\mathbf{z})$ at $\sigma, \sigma^{\prime}= \pm 1$. In addition, it is supposed that $V_{\sigma, \sigma^{\prime}}(0)=0$ and functions $V_{\sigma, \sigma^{\prime}}$ are summable

$$
\sum_{\mathbf{x} \in \mathbb{Z}^{3}}\left|V_{\sigma, \sigma^{\prime}}(\mathbf{x})\right|<\infty
$$

As in the case of statistical model of binary alloy with the nearest neighbors interaction, we introduce (see, for example, [2]) some functions $I, I^{\prime}, I^{\prime \prime}$ on the radius vector $\mathbf{z}$ according to following formulas (this argument is omitted for all functions in them) by means of equations:

$$
V_{1,1}=I+I^{\prime}+I^{\prime \prime}, \quad V_{-1,-1}=I-I^{\prime}+I^{\prime \prime} \quad V_{1,-1}=-I+I^{\prime \prime}
$$

Their solution are

$$
I=\frac{1}{2}\left(\frac{V_{1,1}+V_{-1,-1}}{2}-V_{1,-1}\right), \quad I^{\prime}=\frac{1}{2}\left(V_{1,1}-V_{-1,-1}\right), \quad I^{\prime \prime}=\frac{1}{2}\left(\frac{V_{1,1}+V_{-1,-1}}{2}+V_{1,-1}\right) .
$$

Using introduced functions, the interaction between any two atoms of the alloy is written in the form:

$$
V_{\sigma(\mathbf{x}), \sigma(\mathbf{y})}(\mathbf{z})=I(\mathbf{z}) \sigma(\mathbf{x}) \sigma(\mathbf{y})+\frac{1}{2} I^{\prime}(\mathbf{z})(\sigma(\mathbf{x})+\sigma(\mathbf{y}))+I^{\prime \prime}(\mathbf{z})
$$

Then the hamiltonian (2) takes the following view:

$$
\begin{gathered}
\mathrm{H}[\sigma]=\sum_{\{\mathbf{x}, \mathbf{y}\} \subset \Lambda} I(\mathbf{x}-\mathbf{y}) \sigma(\mathbf{x}) \sigma(\mathbf{y})+\frac{1}{2} \sum_{\{\mathbf{x}, \mathbf{y}\} \subset \Lambda} I^{\prime}(\mathbf{x}-\mathbf{y})(\sigma(\mathbf{x})+\sigma(\mathbf{y}))+\sum_{\{\mathbf{x}, \mathbf{y}\} \subset \Lambda} I^{\prime \prime}(\mathbf{x}-\mathbf{y})= \\
=\sum_{\{\mathbf{x}, \mathbf{y}\} \subset \Lambda} I(\mathbf{x}-\mathbf{y}) \sigma(\mathbf{x}) \sigma(\mathbf{y})+\nu^{\prime} \sum_{\mathbf{x} \in \Lambda} \sigma(\mathbf{x})+\nu^{\prime \prime}|\Lambda|
\end{gathered}
$$

where

$$
\nu=\sum_{\mathbf{z} \in \mathbb{Z}^{3}} I(\mathbf{z}), \quad \nu^{\prime}=\sum_{\mathbf{z} \in \mathbb{Z}^{3}} I^{\prime}(\mathbf{z}), \quad \nu^{\prime \prime}=\sum_{\mathbf{z} \in \mathbb{Z}^{3}} I^{\prime \prime}(\mathbf{z}) .
$$

The thermodynamics of system with the hamiltonian (7) is determined by its specific free energy (here and below, we measure the temperature $T$ by energy units)

$$
F=-\frac{T}{|\Lambda|} \ln Z
$$

with the partition function

$$
Z=\sum_{\{\rho(\mathbf{x})\}} \exp \left(-\frac{1}{T} \mathrm{H}[\sigma]\right)
$$


where the sum is done for all the realization of random sign function $\sigma(\mathbf{x}), \mathbf{x} \in \Lambda$. Respectively, specific internal energy and specific heat (at constant volume) are defined by formulas

$$
U(T)=\frac{\partial(F / T)}{\partial\left(T^{-1}\right)}, \quad C_{V}(T)=\frac{\partial U}{\partial T}=-T^{2} \frac{\partial U}{\partial\left(T^{-1}\right)} .
$$

In the case of the structural order-disorder phase transition, the binary alloy crystal lattice does not rebuilt. Atoms filling its unchanged structure may be located with the presence of spatial ordering or without it. The phase transition consists in that, it is appear a periodic component in the observed averaged value $\langle\rho(\mathbf{x})\rangle$, that is the values

$$
\bar{\rho}_{1}(\mathbf{k})=\lim _{|\Lambda| \rightarrow \infty} \frac{1}{|\Lambda|} \sum_{\mathbf{x} \in \Lambda}\langle\rho(\mathbf{x})\rangle e^{-i(\mathbf{k}, \mathbf{x})}
$$

are nonzero at some wave vectors $\mathbf{k}$ from the inverse crystal lattice.

This function characterize the phase transition, but it is difficult to observe them experimentally. Therefore, it is better to observe the manifestation of phase transition by the so-called structure factor

$$
\bar{\rho}_{2}(\mathbf{k})=\lim _{|\Lambda| \rightarrow \infty} \sum_{\mathbf{x} \in \Lambda}\left(\langle\rho(\mathbf{x}) \rho(0)\rangle-\rho_{0}^{2}\right) e^{-i(\mathbf{k}, \mathbf{x})}
$$

where $\rho_{0}=\bar{\rho}_{1}(0)$. Some peaks appear at values $\mathbf{k}= \pm \mathbf{k}_{0} m / 2 \pi$, if $m$ runs through a finite set.

\section{Lattice gas}

We now introduce occupation numbers $\rho(\mathbf{x})=0,1$ of lattice sites according to formula $\sigma(\mathbf{x})=$ $2 \rho(\mathbf{x})-1$. After that, we express the hamiltonian ( 7$)$ in terms of them:

$$
H[\rho]=\frac{1}{2} \sum_{\mathbf{x}, \mathbf{y} \in \Lambda} \Phi(\mathbf{x}-\mathbf{y}) \rho(\mathbf{x}) \rho(\mathbf{y})-\mu \sum_{\mathbf{x} \in \Lambda} \rho(\mathbf{x})+\varepsilon|\Lambda|
$$

where $2 \nu-\nu^{\prime}=\mu, 4 I(\mathbf{z})=\Phi(\mathbf{z})$, and the additive constant $\varepsilon=\nu^{\prime \prime}-\nu^{\prime} / 2+\nu / 2$ does not contribute a value to the specific heat $C_{V}(T)$, as it will be clear later. The first sum in Eq.(12) is realized independently on sites $\mathbf{x}$ and $\mathbf{y}$ of $\Lambda$. "The interaction potential" $\Phi$ has the property $\Phi(0)=0$ and, owing to Eq.(3), the sum

$$
\sum_{\mathbf{x} \in \mathbb{Z}^{3}}|\Phi(\mathbf{x})|<\infty
$$

is finite.

Substitution of expressions $\nu$ and $\nu^{\prime}$ into the definition of $\mu$ gives the formula

$$
\mu=\sum_{\mathbf{x} \in \mathbb{Z}^{3}}\left(V_{-1,-1}(\mathbf{x})-V_{1,-1}(\mathbf{x})\right) .
$$

It is asymmetric with respect to the replacement of indices 1 and -1 that is a result of asymmetric selection of substitution $\sigma=2 \rho-1$. This choice is done due to the choice of sign $\mu<0$, since, according to the definition of $\mu$, the inequality holds

$$
\sum_{\mathbf{x} \in \mathbb{Z}^{3}} V_{-1,-1}(\mathbf{x})<\sum_{\mathbf{x} \in \mathbb{Z}^{3}} V_{1,-1}(\mathbf{x}) .
$$

If it does not take place, but the inequality

$$
\sum_{\mathbf{x} \in \mathbb{Z}^{3}} V_{1,1}(\mathbf{x})<\sum_{\mathbf{x} \in \mathbb{Z}^{3}} V_{1,-1}(\mathbf{x})
$$

holds, then one should be put $\sigma(\mathbf{x})=1-2 \rho(\mathbf{x})$ and, therefore,

$$
\mu=2 \nu+\nu^{\prime}=\sum_{\mathbf{x} \in \mathbb{Z}^{3}}\left(V_{1,1}(\mathbf{x})-V_{1,-1}(\mathbf{x})\right) .
$$


If Eq.(15) and Eq.(16) are not satisfied simultaneously, then the "order-disorder" phase transition does not occur in the alloy.

The partition function is defined by the following formula on the basis of the hamiltonian (12)

$$
Z=\sum_{\{\rho(\mathbf{x})\}} \exp \left(-\frac{1}{T} \mathrm{H}[\rho]\right)
$$

where the sum is done on all possible realizations $\rho(\mathbf{x})$.

The hamiltonian (12) is defined on the phase space which consists of all two-valued functions $\rho(\mathbf{x})$, i.e. 0 and 1. It defines the system of statistical mechanics, which is called "the lattice gas". In terms of this model, each microscopic state of the alloy is described by the values 0 or 1 , which correspond to the filling of lattice sites by atoms of first and second kinds respectively. In this case, the "order-disorder" phase transition within the framework of such a model corresponds formally to the "liquid-gas" phase transition. Thus, for constructing of a quantitative theory in the framework of equilibrium statistical mechanics which describes thermodynamic behavior of binary alloy and, in particular, describes the "order-disorder" phase transition in it, it is sufficient to solve the problem of thermodynamic behavior of lattice gas. The parameter $\mu$ corresponds to the gas chemical potential.

\section{Construction of the virial expansion}

To calculate the thermodynamic characteristics of binary alloy we apply the so-called virial expansion which is well-known in the gas theory (see, for example, [3]). We write down the hamiltonian (1) in such a form which is similar to that it is studied in this theory. Firstly, for each realization $\rho(\mathbf{x})$, we introduce the set $A \subset \Lambda$ of those sites where it takes the value $1, A=\{\mathbf{x}: \rho(\mathbf{x})=1\}$, we write formulas (12) and (17) in the form

$$
\begin{gathered}
H[A]=-\mu|A|+\sum_{\{\mathbf{x}, \mathbf{y}\} \subset A} \Phi(\mathbf{x}-\mathbf{y}), \\
Z=\sum_{A \subset \Lambda} \exp \left(-\frac{1}{T} \mathrm{H}[A]\right) .
\end{gathered}
$$

Here, the sum is done over all subsets of $\Lambda$. Assuming $A=\left\{x_{1}, x_{2}, \ldots, x_{n}\right\}$, we rewrite the formula (19) in the following form:

$$
Z=\sum_{n=0}^{|\Lambda|} \frac{z^{n}}{n !} \sum_{\mathbf{x}_{1}, \ldots, \mathbf{x}_{n} \in \Lambda}^{\neq} \exp \left(-\frac{1}{T} \sum_{i<j}^{n} \Phi\left(\mathbf{x}_{i}-\mathbf{x}_{j}\right)\right),
$$

where $z=e^{\mu / T}$. Similarly, the sum is done here over all ordered collections $\left\langle\mathbf{x}_{1}, \ldots, \mathbf{x}_{n}\right\rangle$ of points from $\Lambda$ with mismatched components.

In order to present of the partition function (20) in the form analogous to the large partition function which is used in the theory of monatomic gases, we introduce another potential $\tilde{\Phi}(x)$ which is equal to the potential $\Phi(x)$ at all nonzero points $\mathbf{x}$ and $\tilde{\Phi}(0)=\infty$. In terms of this potential, the partition function (20) takes the form:

$$
Z=\sum_{n=0}^{\infty} \frac{z^{n}}{n !} \sum_{\mathbf{x}_{1}, \ldots, \mathbf{x}_{n} \in \mathbb{Z}^{3}} \exp \left(-\frac{1}{T} \sum_{i<j}^{n} \tilde{\Phi}\left(\mathbf{x}_{i}-\mathbf{x}_{j}\right)\right),
$$

that differs from the formula of large partition function of monatomic gas system continuously distributed in a fixed volume only by the fact that, instead of integrals over the atoms radius vectors, the sum (21) is done here.

This circumstance makes it possible immediately to write down the main term of the asymptotical formula of the partition function logarithm in the thermodynamic limit $|\Lambda| \rightarrow \infty$. It is performed by the power series using the so-called Mayer graphs (see, for example, [3])

$$
\frac{1}{|\Lambda|} \ln Z=\sum_{n=1}^{\infty} \frac{z^{n}}{n !} \sum_{\left\langle\mathbf{x}_{1}, \ldots, \mathbf{x}_{n-1}\right\rangle \in \mathbb{Z}^{3}} \Psi_{n}\left(\mathbf{x}_{1}, \ldots, \mathbf{x}_{n}\right)
$$


where $\Psi_{1}(\mathbf{x}) \equiv 1$ and the translational invariant multi-point Meyer functions $\Psi\left(\mathbf{x}_{1}, \ldots, \mathbf{x}_{n}\right)$ are defined by

$$
\Psi_{n}\left(\mathbf{x}_{1}, \ldots, \mathbf{x}_{n}\right)=\sum_{\Gamma_{n}} \prod_{\{i, j\} \in \Gamma_{n}} \tilde{\Psi}\left(\mathbf{x}_{i}-\mathbf{x}_{j}\right) .
$$

Here, $\Psi_{2}(\mathbf{x}, \mathbf{y}) \equiv \tilde{\Psi}(\mathbf{x}-\mathbf{y})$, and the sum is done over all different connected graphs with vertices tagged indices with values from $I_{n} \equiv\{1, \ldots, n\}$ The product is computed over all edges $\{i, j\}$ admissible by the graph $\Gamma_{n}$ in each summand of the sum (23).

As it is known (see, for example, [3]), the logarithm of partition function determines the "pressure" $P$ of lattice gas $P / T=\ln Z /|\Lambda|$ as a function of the parameters $z$ and $T$. In this way, due to the connection of lattice gas model with the statistical system of binary alloy, we have $F=-P$. From this and from the well-known power expansion of pressure (see, for example, [8]), we find that the free energy is determined by Eq.(22) where it is necessary to take the opposite sign.

\section{Specific heat of binary alloy}

In this communication, we present asymptotic formulas of $F / T$ and $C_{V}(T)$ at high temperature region (in the disordered state) or, more strictly, we propose first two terms of the total asymptotic expansion of these physical values. This problem is solved on the basis of the expansion (22). The function $\tilde{\Psi}(\mathbf{x}-\mathbf{y})$ has a "singularity" at $\mathbf{x}=\mathbf{y}$ in the proposed formalism. Namely, assuming $\Psi(\mathbf{x})=\exp (-\Phi(\mathbf{x}) / T)-1$, we have

$$
\tilde{\Psi}(\mathbf{x}-\mathbf{y})=\Psi(\mathbf{x}-\mathbf{y})-\delta_{\mathbf{x}, \mathbf{y}}
$$

where the first term becomes small when $T$ tends to infinity at all values of vectors $\mathbf{x}$ and $\mathbf{y}$ in contrast to the second one. In connection with this situation, to solve the problem, it is necessary such a rearrangement of the series when, firstly, all sums leading to the disappearance of all Kronecker symbols should be produced. These symbols are included in Ursell's functions $\Psi(\mathrm{x}-\mathrm{y})$. At such a rearrangement of the series, we construct the high-temperature expansion of above-mentioned physical values on the basis of Ursell's functions $\Psi(\mathbf{x}-\mathbf{y})$ such that we consider them as small values when $T \rightarrow \infty$. However, we avoid trivial expansions of these functions using series on $1 / T$ powers of them. To solve the problem, we expand the following functions

$$
\tilde{\Psi}_{m+1}\left(X_{m}\right)=\sum_{\Gamma_{m+1}} \prod_{\{i, j\} \in \Gamma_{m+1}}\left(\Psi\left(\mathbf{x}_{i}-\mathbf{x}_{j}\right)-\delta_{\mathbf{x}_{i}, \mathbf{x}_{j}}\right)
$$

according to the degree of Ursell's function $\Psi$ in them.

Let us find the form of coefficients of power expansion in Eq.(22) up to linear occurrence of Ursell's function in them. Since, there is the combinatorial relation

$$
\sum_{\Gamma_{m+1}}(-1)^{l(\Gamma)}=m !(-1)^{m}
$$

$\left(l\left(\Gamma_{m+1}\right)\right.$ is the number of edges in the graph $\left.\Gamma_{m+1}\right)$, then, at zero approximation, we have

$$
\begin{gathered}
\sum_{m=0}^{\infty} \frac{z^{m+1}}{(m+1) !} \tilde{\Psi}_{m+1}^{(0)}\left(X_{m}\right)=\sum_{m=0}^{\infty} \frac{z^{m+1}}{(m+1) !} \sum_{\Gamma_{m+1}}(-1)^{l\left(\Gamma_{m+1}\right)} \prod_{\{i, j\} \in \Gamma_{m+1}} \delta_{\mathbf{x}_{i}, \mathbf{x}_{j}}= \\
=\sum_{m=0}^{\infty}(-1)^{m} \frac{z^{m+1}}{m+1}=\ln (1+z) .
\end{gathered}
$$

To calculate the next approximation, it is sufficient to take into account those graphs $\Gamma_{m+1}$ which may be divided into two connected graphs $\Gamma^{(1)}$ and $\Gamma^{(2)}$. We demand that, at such a division, there is only one edge connecting $\Gamma^{(1)}$ and $\Gamma^{(2)}$ in the graph $\Gamma_{m+1}$. In general case, all such graphs $\Gamma_{m+1}$ give zero contribution into $F$ when summation of corresponding expressions on $X_{m}$ is done. Nonzero contribution of pointed out graphs, which is proportional to first power of Ursell's function, is obtained in such a 
case when Kronecker's symbols correspond to edges in graphs $\Gamma^{(k)}, k=1,2$ and the function $\Psi\left(\mathbf{x}_{j_{1}}, \mathbf{x}_{j_{2}}\right)$ corresponds to the unique edge $\left\{j_{1}, j_{2}\right\}$ connecting them.

Consequently, the part of function $\tilde{\Psi}_{m+1}$ proportional to first power of Ursell's function has the form:

$$
\begin{gathered}
\tilde{\Psi}_{m+1}\left(X_{m}\right)=\sum_{\substack{V_{1}, V_{2} \subset I_{m+1} \\
V_{1} \cap V_{2}=\varnothing, V_{1} \cup V_{2}=I_{m+1}}} \sum_{j_{1} \in V_{1}} \sum_{j_{2} \in V_{2}} \Psi\left(\mathbf{x}_{j_{1}}, \mathbf{x}_{j_{2}}\right) \times \\
\times\left(\sum_{\Gamma^{(1)}}(-1)^{l\left(\Gamma^{(1)}\right)} \prod_{\{j, k\} \in \Gamma^{(1)}} \delta_{\mathbf{x}_{j}, \mathbf{x}_{k}}\right)\left(\sum_{\Gamma^{(2)}}(-1)^{l\left(\Gamma^{(2)}\right)} \prod_{\{j, k\} \in \Gamma^{(2)}} \delta_{\mathbf{x}_{j}, \mathbf{x}_{k}}\right) .
\end{gathered}
$$

After changing of summation variables in this expression, we obtain

$$
\begin{gathered}
\sum_{X_{m} \in\left(\mathbb{Z}^{3}\right)^{m}} \tilde{\Psi}_{m+1}\left(X_{m}\right)=\sum_{X_{m-1} \in\left(\mathbb{Z}^{3}\right)^{m-1}} \sum_{\substack{V_{1}, V_{2} \subset I_{m+1} \\
V_{1} \cap V_{2}=\varnothing, V_{1} \cup V_{2}=I_{m+1}}}\left|V_{1}\right|\left|V_{2}\right| \times \\
\times\left(\sum_{\Gamma^{(1)}}(-1)^{l\left(\Gamma^{(1)}\right)} \prod_{\{j, k\} \in \Gamma^{(1)}} \delta_{\mathbf{x}_{j}, \mathbf{x}_{k}}\right)\left(\sum_{\Gamma^{(2)}}(-1)^{l\left(\Gamma^{(2)}\right)} \prod_{\{j, k\} \in \Gamma^{(2)}} \delta_{\mathbf{x}_{j}, \mathbf{x}_{k}}\right) \sum_{\mathbf{x}_{m} \in \mathbb{Z}^{3}} \Psi\left(\mathbf{x}_{m}\right) \\
=\sum_{\substack{V_{1}, V_{2} \subset I_{m+1} \\
V_{1} V_{2}=\varnothing, V_{1} \cup V_{2}=I_{m+1}}}\left|V_{1}\right|\left|V_{2}\right|\left(\sum_{\Gamma^{(1)}}(-1)^{l\left(\Gamma^{(1)}\right)}\right)\left(\sum_{\Gamma^{(2)}}(-1)^{l\left(\Gamma^{(2)}\right)}\right) \sum_{\mathbf{x}_{m} \in \mathbb{Z}^{3}} \Psi\left(\mathbf{x}_{m}\right) .
\end{gathered}
$$

The summation on all graphs in last expression according Eq.(25) gives

$$
\begin{aligned}
\sum_{X_{m} \in\left(\mathbb{Z}^{3}\right)^{m}} \tilde{\Psi}_{m+1}\left(X_{m}\right)= & \sum_{\substack{V_{1}, V_{2} \subset I_{m+1} \\
V_{1} \cap V_{2}=\mathscr{Q}, V_{1} \cup V_{2}=I_{m+1}}}\left|V_{1}\right| !\left|V_{2}\right| !(-1)^{\left|V_{1}\right|-1}(-1)^{\left|V_{2}\right|-1} \sum_{\mathbf{z} \in \mathbb{Z}^{3}} \Psi(\mathbf{z})= \\
& =m(m+1) !(-1)^{m-1} \sum_{\mathbf{z} \in \mathbb{Z}^{3}} \Psi(\mathbf{z}) .
\end{aligned}
$$

At last, by summation on $m$, from the obtained expression, we find

$$
\sum_{m=1}^{\infty} \frac{z^{m+1}}{(m+1) !} \sum_{X_{m} \in\left(\mathbb{Z}^{3}\right)^{m}} \tilde{\Psi}_{m+1}\left(X_{m}\right)=\sum_{m=0}^{\infty}(-z)^{m} \sum_{\mathbf{z} \in \mathbb{Z}^{3}} \Psi(\mathbf{z})=\left(\frac{z}{1+z}\right)^{2} \sum_{\mathbf{z} \in \mathbb{Z}^{3}} \Psi(\mathbf{z}) .
$$

Consequently, according to Eq.(22) and Eq.(26),

$$
\frac{1}{|\Lambda|} \ln Z=\ln (1+z)+\left(\frac{z}{1+z}\right)^{2} \sum_{\mathbf{x} \in \mathbb{Z}^{3}} \Psi(\mathbf{x}) .
$$

Then, using Eq. (9) and Eq.(11), we obtain that the specific free energy of binary alloy is

$$
\begin{gathered}
F=-T \ln (1+z)-T\left(\frac{z}{1+z}\right)^{2} \psi(T)-\varepsilon, \\
\psi(T)=\sum_{\mathbf{x} \in \mathbb{Z}^{3}} \Psi(\mathbf{x}),
\end{gathered}
$$

the specific internal energy is defined as

$$
U(T)=-\left[\frac{\mu z}{1+z}+\frac{2 \mu z^{2}}{(1+z)^{3}} \psi(T)+\left(\frac{z}{1+z}\right)^{2} \psi^{\prime}(T)\right]+\varepsilon
$$


and the specific heat is represented as

$$
C_{V}(T)=\frac{z T^{2}}{(1+z)^{2}}\left[\mu^{2}+z \psi^{\prime \prime}(T)+\frac{4 \mu z}{1+z} \psi^{\prime}(T)+\frac{2 \mu^{2} z(2-z)}{(1+z)^{2}} \psi(T)\right]
$$

We note that $C_{V}(T)=\frac{\mu^{2}}{4} T^{2}$ at $T \rightarrow \infty$, since $\psi(T) \rightarrow 0$ at $T \rightarrow \infty$ with its derivatives.

\section{References}

1. Kerson Huang, Statistical Mechanics, John Wiley \& Sons, Inc., New York (1963).

2. R.P. Feynman, Statistical Mechanics, Benjamin, Massachussets (1972).

3. J.E. Mayer, M. Geppert-Mayer, Statistical Mechanics, John Wiley \& Sons, New York, (1977).

4. S.N. Vereshchagin, V.A. Dudnikov, L.A. Solovyov, Journal of Siberian Federal University. Chemistry, 9,326$336(2016)$.

5. A.A. Klopotov, A.I. Potekayev, E.V. Kozlov, Yu.I. Tyurin et al., Crystal-geometric and crystal-chemical partterns of emergence of binary and triple compounds on the basis of Ti and Ni, Tomsk State University, Tomsk (2011) [in Russian].

6. A.A. Klopotov, A.I. Potekayev, E.V. Kozlov, V.V. Kulagina, Izvesitiya vyshikh uchebnykh zavedenii. Fizika, 56, $213(2013)$.

7. A.I. Potekayev, V.V. Kulagina, M.D. Starostenkov, A.A. Klopotov, T.N. Markov, M.M. Morozov, Izvesitiya vyshikh uchebnykh zavedenii. Chernaya metallurgia, 6, 65 (2013).

8. D. Ruelle, Statistical mechanics. Rigrous reasults, W.A. Benjamin, Inc., Amsterdam (1969).

9. Yu.P. Virchenko, L.P. Danilova, Belgorod State University Scientific Bulletin. Mathematics 63 Physics, 46, 138 (2017). 